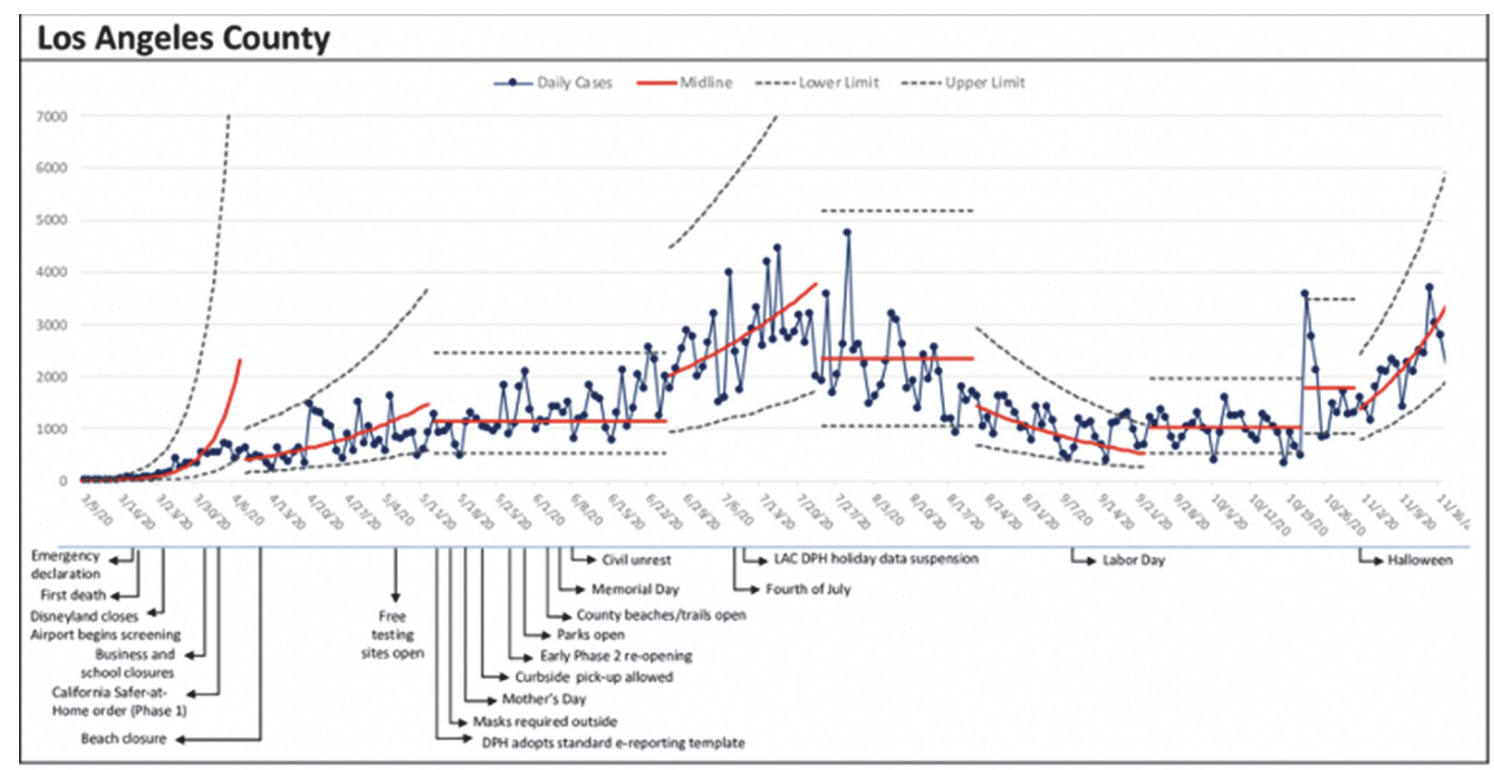

Abstract 13 Figure 5 Hybrid C Chart and I Chart: COVID-19 case counts

student or staff member, and vaccination drawing from community-level data linked with school region (figures 3 and 4). Data displays showed variation in actionable formats.

Conclusions Common data displays in COVID-19 lack elements required to learn from variation, including denominators, disaggregation (granularity), and operationally relevant stratification (such as subregion and school configuration). Providing these displays offered actionable data to the school district that they employed in areas such as vaccination outreach and testing protocols. There is an ongoing need for scientific input and support of actionable data displays, for use by public health as well as school districts. Future work will include incorporating these displays into operations and generating them from developing databases.

\section{ADDRESSING SOCIAL HEALTH AND EARLY CHILDHOOD WELLNESS: NATIONAL RESILIENCE LEARNING COLLABORATIVE}

${ }^{1}$ Marian Earls, Megan Heavrin, ${ }^{2}$ Eileen Reilly. ${ }^{1}$ Marian F Earla Consulting; ${ }^{2}$ American Academy of Pediatrics

\subsection{6/bmjoq-2021-IHI.14}

Background Unmet social needs in early childhood create risk of lifelong physical/mental health challenges. Pediatricians can intervene by routinely assessing child development including child and family social emotional wellbeing. Many providers report barriers to interventions that meet families' social needs and lack reimbursement.

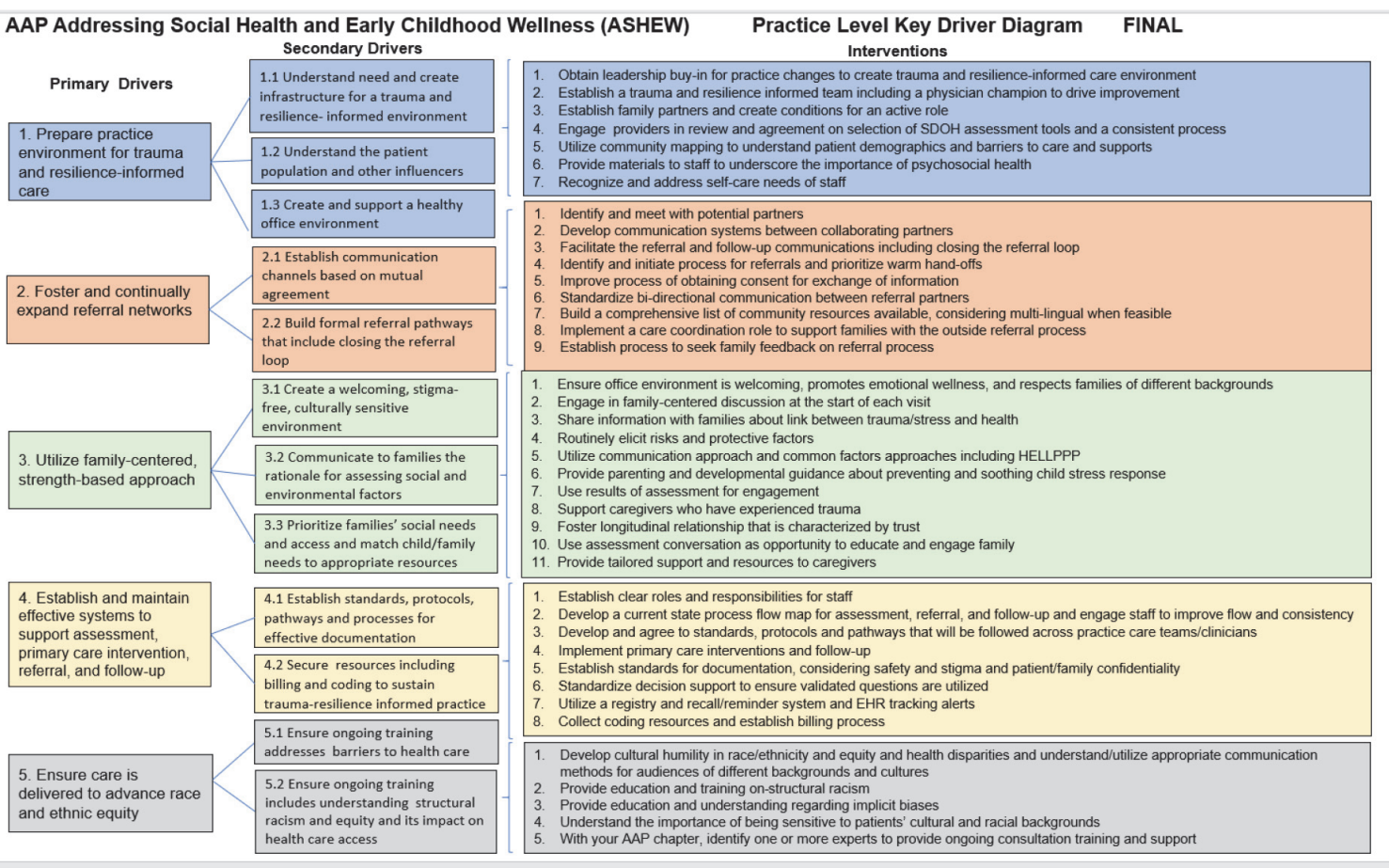

Abstract 14 Figure 1 AAP Addressing Social Health and Early Childhood Wellness (ASHEW) key driver diagram 
Key:

Goal $90 \% 1,2,3$

10-Point Median

Nonrandom Variation

Shift: 6 consecutive points above median
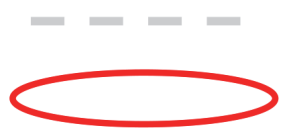

1. Family Strengths/Protective Factors

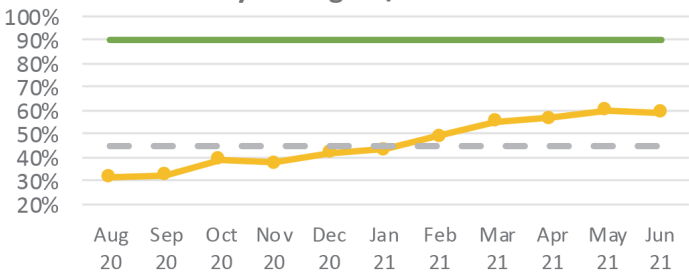

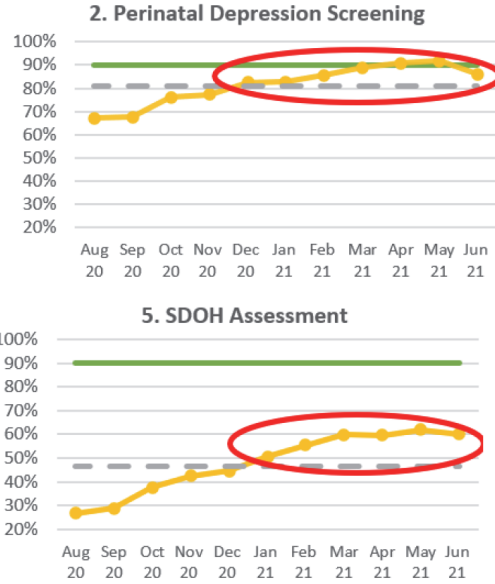

8. Social Emotional Development

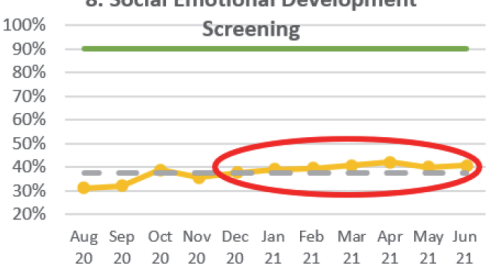

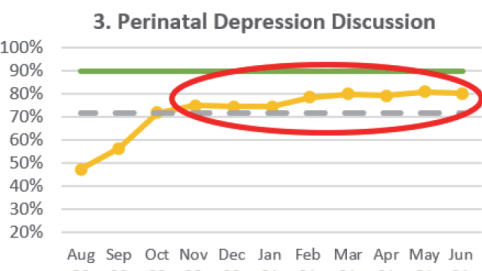

$\begin{array}{lllllllllll}20 & 20 & 20 & 20 & 20 & 21 & 21 & 21 & 21 & 21 & 21\end{array}$
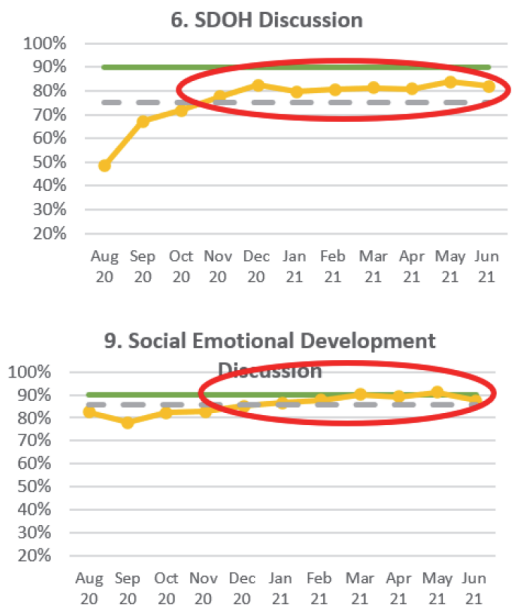

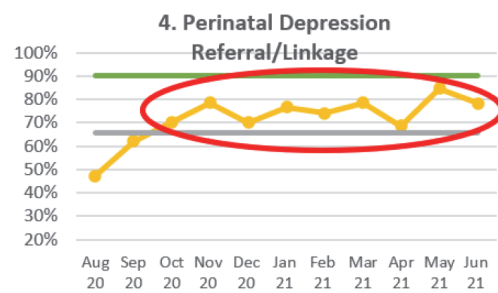

7. SDOH Referral/Linkage

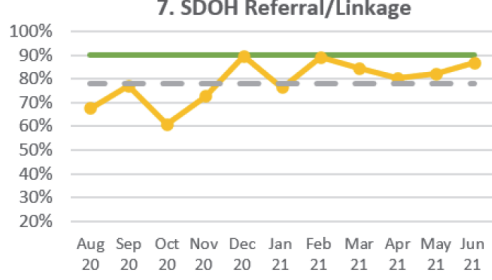

10. Social Emotional Development

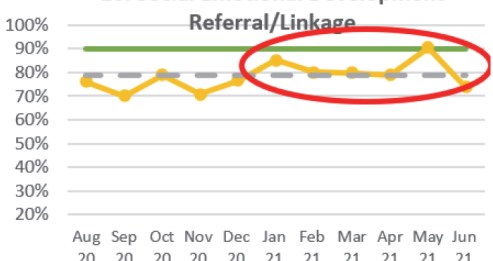

\begin{abstract}
${ }^{1} 10$ charts or all available were reviewed at the beginning of each month using the previous month's well child checks for ages 6-, 15-, 24-, and 48-months. Ages chosen based on the number of recommended screens at each visit (eg, developmental, autism spectrum disorder) to reduce burden on clinicians.

2 Includes at least one documented perinatal depression screen at the 1-, 2-, 4-, and 6-month visits.

${ }^{3}$ Chart data for cycle $1186 \%$ complete, $n=57$
\end{abstract}

Abstract 14 Figure 2 ASHEW run charts. ${ }^{1} 10$ charts or all available were reviewed at the beginning of each month using the previous month's well child checks for ages 6-, 15-, 24-, and 48-months. Ages chosen based on the number of recommended screens at each visit (e.g., developmental, autism spectrum disorder) to reduce burden on clinicians. ${ }^{2}$ Includes at least one documented perinatal depression screen at the 1-, 2-, 4-, and 6-month visits. ${ }^{3}$ Chart data for cycle $1186 \%$ complete, $n=57 ;{ }^{2}$ Includes at least one documented perinatal depression screen at the 1-, 2-, 4-, and 6-month visits; ${ }^{3}$ Chart data for cycle $1186 \%$ complete, $n=57$

Objectives Test a national strategy to increase social needs assessment, family-centered discussion, referral/linkage, and follow-up with families at $90 \%$ of $0-5$ years well child checks (WCC).

Methods The American Academy of Pediatrics (AAP) conducted an 11-month quality improvement (QI) virtual learning collaborative using the Model for Improvement to address social needs (figure 1). Seven AAP chapters, supported by AAP, provided 66 primary care practices monthly education/ collaboration, data review, and coaching. Practice teams included a physician leader, staff, administrative support, and family advisor. Practices conducted monthly chart reviews $(\mathrm{N}=40)$ following 6-month WCC for perinatal depression (PD), and following 6, 15, 24, and 48-month WCC for social drivers of health (SDOH) and social emotional development (SED). Chart reviews assessed (yes/no) documentation of screening, discussion, and referral/linkage. Improvements from baseline were examined using run charts. Practice transformation strategies included family engagement during WCCs, embedded family advisors on practice QI teams, strengthened community partnership, and enhanced support from AAP chapter family advisors. Quarterly qualitative surveys further assessed practice transformation on trauma and resilience informed care, family-centered approaches, and care to advance race and ethnic equity.

Results Chart review data shows nonrandom variation in 8 of 10 measures or a shift of 6 consecutive points above the median (figure 2).

Conclusions Pediatric providers can make quality improvements to address unmet social needs using early relational health to foster family resilience. Next steps include continued progress toward goals during a second phase and enhanced training resources shared with all pediatric providers to increase rates of screening, discussion, referral/linkage, and ascertaining outcomes from families. 